\title{
Pathological mechanism of hidden blood loss after TKA: oxidative stress induced by free fatty acids
}

Tao Yuan ( $\square$ yuant1205@163.com )

Jinling Hospital

Xin Yu

Nanjing University Medical School

Hong Qian

East Region Military Command General Hospital

Jia Meng

East Region Military Command General Hospital

Hui Jiang

East Region Military Command General Hospital

Shaoqiang Yang

Southern Medical University

Nirong Bao

East Region Military Command General Hospital

Jianning Zhao

East Region Military Command General Hospital

\section{Research article}

Keywords: hidden blood loss, free fatty acids, reactive oxygen species, total knee arthroplasty

Posted Date: April 1st, 2020

DOI: https://doi.org/10.21203/rs.3.rs-19898/v1

License: (c) (1) This work is licensed under a Creative Commons Attribution 4.0 International License.

Read Full License 


\section{Abstract}

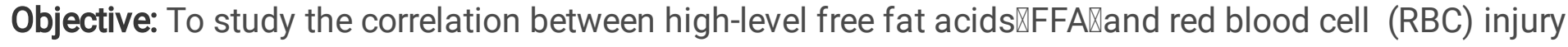
and to explore the pathological mechanism of hidden blood loss (HBL) after total knee arthroplasty (TKA).

Methods: Perioperative blood indexes were tested in 120 patients underwent unilateral total knee replacement for end-stage knee osteoarthritis. Venous blood samples were collected before operation and $24 \mathrm{~h}, 48 \mathrm{~h}, 72 \mathrm{~h}$ and $120 \mathrm{~h}$ after operations. The changes of FFA, hemoglobin $(\mathrm{Hb})$ and red blood cell number in the blood samples were detected by automatic hematology analyzer. The activity of glutathion peroxidase (GSH-Px), total superoxide dismutase (T-SOD) and hydrogen peroxide $\left(\mathrm{H}_{2} \mathrm{O}_{2}\right)$ levels in the blood were measured. Measurement of reactive oxygen species $₫ \mathrm{R} O S \bigotimes$ was performed by flow cytometry. Meanwhile, the morphological changes of RBC were analyzed under microscope.

Results: Significant hidden blood loss was observed for all patients. The $\mathrm{Hb}$ content, RBC and hematocrit \Hct \decreased significantly $24 \mathrm{~h}$ after surgery $(\mathrm{P}<0.05)$ \while FFA concentration was significantly increased and heteromorphic red blood cells appeared under the microscope. The hemoglobin content decreased to the lowest level at $48 \mathrm{~h}$ after the operation $(P<0.01)$. The change of $H B L$ was the most significant according to the Gross equation with the levels of FFA and ROS in the blood increased significantly and reached the peak at $48 \mathrm{~h}$ after operation $(\mathrm{P}<0.01)$. Meanwhile, GSH-PX activity, T-SOD activity and $\mathrm{H}_{2} \mathrm{O}_{2}$ levels significantly decreased compared with preoperative tested samples $(P<0.01)$. Microscopically, erythrocyte atypia increased significantly with cellular rupture and necrosis identified. After $72 \mathrm{~h}$ of operation, ROS concentration began to decline along with FFA concentration. However, the $\mathrm{Hb}$ and RBC began to rise. Also, GSH-Px activity, T-SOD activity and $\mathrm{H}_{2} \mathrm{O}_{2}$ levels increased as well. All tested parameters tended to return to normal levels five days after surgery.

Conclusion: High levels of FFA in blood can induce oxidative stress and damage RBCs, which in turns causes HBL after surgery.

\section{Introduction}

Hidden blood loss often occurs after traumatic fracture or surgery, which directly results in increased blood loss and prolonged recovery time and hospital stay $[1,2]$. There are many hypotheses about hidden blood loss, but the pathological mechanism is still not clear. As early as 1973, Pattison et al. found that there was anemia inconsistent with intraoperative bleeding after knee replacement, and speculated that there was invisible blood loss [3]. Sehat et al. first proposed the concept of HBL in 2000 and suggested that the HBL accounted for about $50 \%$ of the total blood loss through the Gross equation. Their research pointed out that the average perioperative blood loss of patients with knee arthroplasty was about $1474 \mathrm{ml}$, while the HBL was as high as $735 \mathrm{ml}[4]$.

During TKA, there are two noteworthy operations. The first action is engaging a drill alignment guide to the femoral component between the femoral condyles and drill a longitudinal channel through the 
femoral aperture and into the femur. The second operation is engaging a drill alignment guide to the tibial component and extending the tibial aperture by drilling a longitudinal channel through the tibia aperture and into the tibia. Both of these procedures will increase the pressure in the medullary cavity, where a large number of lipomicron may enter the circulation under high pressure [5-7].

FFA is a catabolic product of lipomicron, which is composed of oleic acid, palmitic acid, linoleic acid, etc., and can be combined with albumin to exist in the blood [8]. The concentration of free fatty acids in serum is related to lipid metabolism, sugar metabolism and endocrine function. Studies have shown that high concentrations of FFA can induce oxidative stress and increase the production of ROS [9]. Active oxygen free radicals are directly or indirectly converted from oxygen and its derivatives. It can react with polyunsaturated fatty acids on the cell membrane to generate peroxides that have a toxic effect on the cells, resulting in protein oxidation, damage of DNA mutation, lipid oxidation, cell foaming. Oxidative damage of lipids in the erythrocyte membrane can directly lead to the damage of red blood cells [10-12]. In this study, we examined the perioperative blood parameters of knee replacement patients to study the relationship between FFA and HBL and explored the pathological mechanism of HBL.

\section{Method And Materials}

The prostheses used by all patients were EMINI MK囚 full-anatomical rotary platforms produced by LINK, Germany. Blood smear prepared by Wright staining kit (Hongbokang pharmaceutical technology, China); Polarized light microscope (NIKON ECLIPSE 501, Japan); hematology analyzer (SYSMEX XE-5000, Japan); ROS release was detected with ROS Detection Kit (Beyotime Institute of Biotechnology, China); The $\mathrm{H}_{2} \mathrm{O}_{2}$ concentration and GSH-Px and SOD activities were determined with commercially available assay kits (Nanjing Jiancheng Bioengineering Institute, China); Centrifuge (Hermle Universal Centrifuge Z323, Germany).

\section{Patient selection}

This study conducted a prospective study of 120 patients who received TKA in Jinling from April 2019 to December 2019; There were 55 males (45.8\%) and 65 females (54.2\%), with an average age of 69 years (range 58-78 years).

Inclusion criteria: $₫$ All patients undergoing aseptic knee osteoarthritis with clinical manifestations of knee pain, varus deformity and limited daily life, which were ineffective after conservative treatment and had no other basic diseases; $\nabla$ Objective, actual, accurate, timely and complete perioperative medical records and the blood samples collected met the measurement requirements; $\nabla$ The patient gave informed consent to the trial scheme.

Exclusion criteria: $₫$ Preoperative blood tests suggest anemia and perioperative blood transfusion patients; Preoperative blood tests suggest anemia, perioperative blood transfusion patients; $\mathbb{\otimes}$ Patients with excessive postoperative activity, obesity, poor surgical tolerance and patients with severe diabetes and cardiopulmonary insufficiency; $\otimes$ Patients with hyperlipidemia, preoperative anemia or hematological 
diseases; $\otimes$ Patients with cerebral infarction or combined with varicose veins of lower limbs 3 months before surgery; $\otimes$ Patients with liver or kidney disease or dysfunction.

\section{Method}

\section{Perioperative management of TKA}

All operations were performed by the same chief physician and the perioperative management was performed by the same attending physician. Patient's height, weight, body mass index, physical examination and vital signs were well recorded before the operation. All patients were routinely given antibiotics to prevent infection $0.5 \mathrm{~h}$ before surgery; During the operation, a tourniquet was used (the tourniquet pressure is subject to the disappearance of the dorsal foot artery fluctuations); In combination with epidural anaesthesia, the operation time is 45-62 minutes (53.6 minutes in average). Adequate intraoperative hemostasis and the tibial plateau and femur osteotomy were based on the principle of restoring force lines, balancing soft tissues and restoring the patella trajectory; After the operation, a drainage tube was placed with elastic bandage applied to bandage the affected limb with moderate pressure from the ankle to the middle thigh. $24 \mathrm{~h}$ after the operation, deep vein thrombosis was prevented by oral anticoagulant rivaroxaban (Bayer) and the use of a lower limb venous thrombotherapy device; At the same time, positive instructed the balance of motion and quiescence had better effect on the Function Rehabilitation and ROM of knee joint. The drainage tube was removed within 48h. During the perioperative period, the homeostasis was maintained according to the fluid intake and outflow.

\section{Evaluation of blood test}

Blood samples were collected preoperative and $24 \mathrm{~h}, 48 \mathrm{~h}, 72 \mathrm{~h}$ and $120 \mathrm{~h}$ after surgery. Samples were placed in heparin anticoagulant tubes and mixed for backup. Appropriate blood samples were stained with Wright's stain to prepare erythrocyte blood smears. Then the morphology of red blood cells was observed under polarized light microscope. Other samples were submitted for inspection within two hours and the RBC, Hct, $\mathrm{Hb}$ content, and FFA concentration were measured by a fully automatic hematology analyzer system. The oxidative stress related indicators $\mathrm{GSH}-\mathrm{Px}, \mathrm{H}_{2} \mathrm{O}_{2}$ and T-SOD were measured by the kit-guided method: GSH-Px activity was measured by fluorescence assay, T-SOD activity was measured by xanthine oxidase assay, and $\mathrm{H}_{2} \mathrm{O}_{2}$ content change was measured by spectrophotometry. ROS release was detected with ROS Detection Kit.

\section{Calculation of blood loss}

Visible blood loss (VBL) mainly includes intraoperative blood loss (negative pressure aspirator retrieves blood, intraoperative gauze), postoperative drainage volume (drainage tube drainage).

The patient estimated blood volume ( EBV ) can be calculated by using the formula [4]区

EBV $=k_{1} \times$ height $\left(m^{3}\right)+k_{2} \times$ weight $(k g)+k_{3}$ 
where $\mathrm{k}_{1} 0.3669, \mathrm{k}_{2} 0.03219, \mathrm{k}_{3} 0.6041$ for men; and $\mathrm{k}_{1} 0.3561, \mathrm{k}_{2} 0.03308, \mathrm{k}_{3} 0.1833$ for women.

Calculated blood loss (CBL) can be calculated by Gross formula: CBL= EBV $\left(\mathrm{H}_{0}-\mathrm{H}_{1}\right) / \mathrm{Hav}$

where, $H_{0}$ is preoperative $H c t, H_{1}$ is postoperative $H c t$, $H a v=\left(H_{0}-H_{1}\right) / 2$ is mean value of hematocrit preoperative and postoperative.

As transfusion patients were excluded from our study, the $\mathrm{CBL}$ can be considered as total blood loss (TBL), So we can figure out: $\mathrm{HBL}=\mathrm{CBL}-\mathrm{VBL}$

\section{Statistical analysis}

All calculations and statistical analyses were performed using the SPSS version 19.0 software. Values were expressed as mean standard deviation and analyzed by one-way analysis of variance followed by Dunnett's t-test. In all cases, $\mathrm{P}<0.05$ was regarded as statistically significant.

\section{Results}

Among the 120 patients involved in the study, 1 patient developed deep vein thrombosis in the lower extremity after surgery and 9 patients received blood transfusion during the perioperative period. Therefore, a total of 110 patients were brought into the result analysis including 54 males and 56 females. All patients had completed relevant examination data. The height of the patients was $168.3 \pm$ $9.5 \mathrm{~cm}$ and the average weight was $67.5 \pm 15.3 \mathrm{~kg}$. The average Hct before operation was $35.2 \pm 3.5 \%$, while it was $27.4 \pm 2.8 \%$ after operation; According to the intraoperative blood loss and postoperative drainage volume, the visible blood loss was $478 \pm 126 \mathrm{~mL}$, according to the Gross formula, the total blood loss was $1036 \pm 128 \mathrm{ml}$, and the hidden blood loss was $565 \pm 72 \mathrm{ml}$. Our findings suggest that $\mathrm{HBL}$ accounts for about $54.5 \%$ of total blood loss.

The average $\mathrm{Hb}$ value of the patients before surgery was $132.5 \pm 11.7 \mathrm{~g} / \mathrm{L}$, and the RBC was $(4.18 \pm 0.45)$ $\times 10^{12} / \mathrm{L}$. $24 \mathrm{~h}$ after surgery. The $\mathrm{Hb}$ and RBC were significantly reduced to $105.5 \pm 8.3 \mathrm{~g} / \mathrm{L}$ and $(3.70 \pm$ 0.38) $\times 10^{12} / \mathrm{L}$. According to the decline of Hct combined with the Gross formula, it can be found that $\mathrm{HBL}$ had occurred. $\mathrm{Hb}$ and RBC continued to decline after surgery, and decreased to the lowest value after about 48 hours, dropping to $85.5 \pm 7.5 \mathrm{~g} / \mathrm{L}$ and $(2.75 \pm 0.30) \times 10^{12} / \mathrm{L}$, respectively. Gross formula results indicated that the hidden blood loss reached the peak value $72 \mathrm{~h}$ later. $\mathrm{Hb}$ and RBC gradually recovered and these indicators took turn for the better 5 days after operation (Fig. 1).

The concentration of FFA was $0.32 \pm 0.11 \mathrm{mmol} / \mathrm{L}$ before surgery and increased to $(0.95 \pm 0.26) \mathrm{mmol} / \mathrm{L}$ $24 \mathrm{~h}$ after the operation, which was almost three times higher than that before surgery. The content of ROS also increased significantly as well. $48 \mathrm{~h}$ later, FFA concentration was as high as $1.28 \pm 0.33 \mathrm{mmol} /$ $L$ and ROS test results indicated that it was also significantly increased. Meanwhile, the alteration of HBL was also the most obvious (Fig. 2). The levels of FFA gradually declined $72 \mathrm{~h}$ after surgery and tended to return to normal levels after 5 days. The above results suggested that FFA and ROS levels increased 
significantly $48 \mathrm{~h}$ after the operation, and the changes trend to synchronize with $\mathrm{HBL}$, which indicated that FFA was closely related to HBL (Fig. 3). Moreover, the increased ROS suggested that the pathogenesis mechanism of HBL may be associated with oxidative stress response.

What's more, indicators related to the redox reaction showed that GSH-Px activity, T-SOD activity and $\mathrm{H}_{2} \mathrm{O}_{2}$ content declined significantly $24 \mathrm{~h}$ after surgery. In addition, erythrocyte atypia was observed under the microscope, which was mainly characterized by crenated erythrocyte. This phenomenon continued to aggravate, GSH-Px activity, T-SOD activity and $\mathrm{H}_{2} \mathrm{O}_{2}$ content were decreased to the minimum $48 \mathrm{~h}$ later, and the erythrocyte atypia also increased significantly. From the microscopic viewpoint, there were many red blood cells with spindle and oblong shapes, pleomorphism, shrinkage, deformation, rupture, and breaking (Fig. 4). After $72 \mathrm{~h}$, the situation began to improve, GSH-Px activity, T-SOD activity, and $\mathrm{H}_{2} \mathrm{O}_{2}$ content increased, and red blood cell aberrations decreased (Fig. 5-7).

\section{Disscussion}

In this study, we confirmed the existence of HBL by examining and analyzing the perioperative blood indexes of patients with knee arthroplasty, and the results showed that the total perioperative blood loss was $1036 \mathrm{ml}$ and significantly lower than the earlier reported $1474 \mathrm{ml}$, suggesting that the improvement of perioperative management could reduce the total blood loss and visible blood loss. But the proportion of $\mathrm{HBL}$ is $54.5 \%$, which is higher than the $50 \%$ reported previously [4], indicating that the control and management of $\mathrm{HBL}$ is crucial.

There are many hypotheses about HBL. Some studies had suggested that HBL may be due to partial hemolysis caused by blood transfusion. However, clinical studies had found that there was no difference in HBL between the transfusion group and non-transfusion group [13, 14]. However, in order to eliminate potential interference, perioperative blood transfusion patients were not included in the study. It is reported that the use of tourniquet during TKA can increase the risk of HBL. The study divided the patients into the tourniquet group and non- tourniquet group, and the results showed that there was no significant difference in operation time between the groups, and the visible blood loss in the nontourniquet group increased about $180 \mathrm{ml}$ compared with the tourniquet group. Instead, the HBL was about $16 \%$ lower than that in the tourniquet group. However, there was still significant HBL in the nontourniquet group that could not be explained $[15,16]$. In addition, the " third mesooecium" theory also partially explained the HBL. Boswell et al. believe that the HBL occurs after TKA due to tissue blood oozing and fluid oozing into the surrounding tissue clearance (i.e., the third space) $[17,18]$. Therefore, after the surgery, we routinely use elastic bandages to bandage the affected limb from the ankle to the middle of the thigh and pressurize appropriately to reduce bleeding, and applied a negative pressure drainage ball to drain the hematocele or hydrops in the joint cavity. With these measures, it was difficult for the blood to penetrate into the surrounding tissue space, and the amount of HBL triggered by this way was limited. 
In the previous study, we successfully constructed the animal model of HBL by injecting linoleic acid and arachidonic acid into rats. Linoleic acid and arachidonic acid are important components of FFA. Our study found that when the blood linoleic acid or linolenic acid reached a certain concentration, it would induce oxidative stress response, resulting in the destruction of red blood cells and HBL [19-21].

HBL often occurs after joint replacement and traumatic fracture surgery, their common feature is that a large amount of fat droplets leak out of the medullary cavity, and penetrate deep into the circulation, tissues and cells; Especially during joint replacement, the manipulation of the femur and tibia can increase the pressure of the medullary cavity and force the fat particles into the blood circulation $[6,7,22$, 23]. The effects of its metabolites on tissue cells need further study. Our results showed that the concentration of FFA in the blood increased significantly $48 \mathrm{~h}$ postoperatively, indicating that large amounts of small fat droplets into the bloodstream during the operation, and the RBC and $\mathrm{Hb}$ were significantly reduced; At the same time, HBL was also the most obvious, and a quantity of damaged red blood cells can be found under the microscope. $72 \mathrm{~h}$ after the operation, with the catabolic metabolism of fat particles, FFA concentration decreased significantly, and erythrocyte and hemoglobin level gradually increased. Meanwhile, the morphology of red blood cells began to improve under the microscope, and the number of erythrocytes with atypia decreased gradually. This indicates that FFA, a metabolite of small fat droplets, is closely related to the reduction and destruction of red blood cells after surgery.

Studies have pointed out that the FFA can help to induce the generation of ROS in endothelial and vascular smooth muscle cells [24]. High concentration of FFA stimulation can increase the production of highly reactive molecular oxygen clusters and reactive nitrogen clusters, which triggers oxidative stress, prolonged imbalance between the production of highly reactive molecules and anti-oxidant effects resulting in tissue damage. These active molecules can directly oxidize and damage DNA, proteins and lipids, and can also act as functional molecular signals, activating a variety of stress-sensitive signaling pathways in cells, which are closely related to insulin resistance and impaired $\beta$-cell function $[25,26]$. Studies have shown that high levels of FFA lead to a large amount of ROS production and oxidative stress, which can also activate stress-sensitive signaling pathways. As fatty acid synthase (FAS) genes activate Akt and sterol regulatory element binding protein-1 under hypoxic conditions Up-regulated, so FAS expression level is associated with hypoxia in the body. It is worth noting that Akt is also upregulated by $\mathrm{H}_{2} \mathrm{O}_{2}$; Under hypoxic conditions, the level of FAS protein is significantly increased as well as the production of ROS in cells $[27,28]$. It suggests that the expression of FAS gene is actively controlled by hypoxia, which is also related to the amount of ROS in cells. During knee arthroplasty, the use of tourniquets can lead to poor blood supply and tissue hypoxia in the affected limb over a period of time, and local tissue swelling and reduced activity after operation also exacerbate blood and tissue hypoxia. As the concentration of fat droplets in blood and tissues increased after surgery, the FAS required for metabolism increases. Therefore, blood and tissue hypoxia will accelerate the catabolic metabolism of $\mathrm{H}_{2} \mathrm{O}_{2}$ in the blood. As a consequence, the level of $\mathrm{H}_{2} \mathrm{O}_{2}$ in the blood decreased significantly $24 \mathrm{~h}$ and $48 \mathrm{~h}$ after surgery. High concentration of FFA will induce the production of ROS in vascular smooth muscle cells, the results of flow cytometry showed that ROS levels increased significantly after 48 hours, which 
may lead to an imbalance of redox reaction. Free radicals can damage cells by acting on polyunsaturated fatty acids on cell membranes, exacerbating oxygen free radical reactions, and lipid peroxidation, the intermediates of lipid peroxidation can react with membrane proteins to polymerize and cross-link proteins. In addition, the carbonyl products of lipid peroxidation (such as malondialdehyde) can also attack the amino groups of membrane protein molecules, resulting in intramolecular and intermolecular cross-linking of proteins. On the other hand, free radicals can also covalently bind directly to enzymes or receptors on the membrane [29-32]. These oxidations damage the spatial configuration of enzymes, receptors and ion channels embedded in the membrane system, destroying the integrity of the membrane and affecting the function of the membrane and antigen specificity, which eventually leads to the cell damage and lesions. Therefore, the oxidative stress induced by FFA can destroy red blood cells and result in erythropenia and HBL.

SOD is an antioxidant metal enzyme in body. It can catalyze the disproportionation of superoxide anion radicals to generate oxygen and $\mathrm{H}_{2} \mathrm{O}_{2}$, which plays a crucial role in the balance between oxidation and antioxidant activity in the body. So, SOD is closely related to the occurrence and development of many diseases [32]. The activity of total superoxide dismutase (T-SOD) can reflect the ability of the body to remove oxygen free radicals [33]. Glutathione peroxidase (GSH-Px) is an important peroxidase widely found in the body. The active center of GSH-Px is selenocysteine, and its activity can reflect the selenium level of the body. Selenium, as a component of the GSH-Px enzyme system, can catalyze the transformation of GSH into GSSG, and reduce toxic peroxides to non-toxic hydroxyl compounds so as to protect the structure and function of cell membranes from the interference and damage of oxides [3435]. The decrease of NADPH was linearly related to the activity of GSH-Px. The physiological function of $\mathrm{GSH}-\mathrm{Px}$ in plasma is mainly to catalyze GSH, participate in the peroxidation reaction, remove peroxides and hydroxyl radicals generated during cellular respiratory metabolism, thereby reducing the peroxidation of polyunsaturated fatty acids in the cell membrane $[19,36]$. Studies have shown that FFA can stimulate neutrophils to produce $\mathrm{H}_{2} \mathrm{O}_{2}$ and hypochlorous acid, which can oxidize and deplete SOD and GSH-Px on the surface of the cell membrane [37,38]. In our study, with the increase of FFA concentration in blood, the activities of T-SOD and GSH-Px decreased significantly, and the morphology of red blood cells also showed obvious cytomembrane destruction, suggesting that the lipid droplet metabolite FFA in the blood induced a series of oxidation reactions in the body. The imbalance of the redox reaction leads to the destruction and significant reduction of red blood cells. Accompanied by the metabolism of FFA and the disappearance of oxidative stress stimuli, the $\mathrm{Hb}$ and RBC increased by self-compensation, the activities of T-SOD and GSH-Px return to equilibrium.

\section{Conclusion}

In summary, high level of FFA can induce oxidative stress in vivo and damage red blood cells, which eventually leads to HBL; It suggests that intraoperative clearance of fat droplets in the medullary cavity and the operative area, and the use of antioxidants postoperative may reduce the occurrence of HBL. 


\section{Abbreviations}

HBL: Hidden Blood Loss

TKA: Total Knee Arthroplasty

T-SOD: Total Superoxide Dismutase

GSH-Px: Glutathione peroxidase

\section{Declarations}

\section{Ethics approval and consent to participate}

The study adhered to the Declaration of Helsinki and was approved by the National Regional Committee for Medical and Health Research Ethics, and registered with the Ethics Committee of Jinling Hospital (2019NZGKJ-006). Written informed consent was obtained from all participants prior to any study-related procedure.

\section{Availability of data and materials}

The datasets used during the current study are available from the corresponding author.

\section{Competing interests}

The authors declare that they have no competing interests.

\section{Funding}

This study was supported by the National Natural Science Foundation of China (81772318) and Natural Science Foundation of Jiangsu Province (BE2017023)

\section{Authors' contributions}

B.N.R. and Z.J.N. contributed to the ideas and final version for this commentary; Y.T contributed to analyze data and draft manuscript, Y.X, Q.H, M.J, and J.H contributed to collect and test the blood samples, Y.S.Q and L.Z.D contributed to collect and record patient information. 


\section{Acknowledgments}

We thank Ting Wang from State Key Laboratory of Bioelectronics, Southeast University, Nanjing, China.

\section{References}

1. Smith, G. H., Tsang, J., Molyneux, S. G., \& White, T. O. (2011). The hidden blood loss after hip fracture. Injury, 42(2), 133-135.

2. Foss, N. B., \& Kehlet, H. (2006). Hidden blood loss after surgery for hip fracture. The Journal of bone and joint surgery. British volume, 88(8), 1053-1059.

3. Pattison, E., Protheroe, K., Pringle, R. M., Kennedy, A. C., \& Dick, W. C. (1973). Reduction in haemoglobin after knee joint surgery. Annals of the rheumatic diseases, 32(6), 582.

4. Sehat, K. R., Evans, R., \& Newman, J. H. (2000). How much blood is really lost in total knee arthroplasty: correct blood loss management should take hidden loss into account. The Knee, 7(3), 151-155.

5. Yager, E. R., Cronin, S. R., Howell, S. M., \& Willard, D. B. (2019). U.S. Patent Application No. $16 / 162,520$.

6. Lu, K., Xu, M., Li, W., Wang, K., \& Wang, D. (2017). A study on dynamic monitoring, components, and risk factors of embolism during total knee arthroplasty. Medicine, 96(51).

7. Bao, N., Zhou, L., Cong, Y., Guo, T., Fan, W., Chang, Z., \& Zhao, J. (2013). Free fatty acids are responsible for the hidden blood loss in total hip and knee arthroplasty. Medical hypotheses, 81(1), 104-107.

8. Sztefko, K., \& Panek, J. (2001). Serum free fatty acid concentration in patients with acute pancreatitis. Pancreatology, 1(3), 230-236.

9. Chen, Q., Su, Y., Ju, Y., Ma, K., Li, W., \& Li, W. (2018). Astragalosides IV protected the renal tubular epithelial cells from free fatty acids-induced injury by reducing oxidative stress and apoptosis. Biomedicine \& Pharmacotherapy, 108, 679-686.

10. Srivastava, S., Singh, D., Patel, S., \& Singh, M. R. (2017). Role of enzymatic free radical scavengers in management of oxidative stress in autoimmune disorders. International journal of biological macromolecules, 101, 502-517.

11. Banks, M. A. (2017). Vitamin E and polyunsaturated fats: antioxidant and pro-oxidant relationship. In Oxidants, Antioxidants and Free Radicals (pp. 95-111). Routledge.

12. Cortelazzo, A., De Felice, C., Guerranti, R., Leoncini, R., Barducci, A., Leoncini, S., ... \& Armini, A. (2015). Erythrocyte cytoskeletal-plasma membrane protein network in Rett syndrome: effects of $\omega-3$ polyunsaturated fatty acids. Current Proteomics, 12(4), 217-226.

13. Huerfano, E., Huerfano, M., Shanaghan, K. A., \& Della Valle, A. G. (2019). Topical tranexamic acid in revision total knee arthroplasty reduces transfusion rates and may be associated with earlier 
recovery. The Journal of arthroplasty, 34(7), S249-S255.

14. Hu K Z, He-Yan S, Sui C. Effects of five treatment regimens on blood loss and blood transfusion in total knee arthroplasty: a preliminary study in China[J]. International journal of clinical pharmacology and therapeutics, 2017, 55(5): 433.

15. Tetro, A. M., \& Rudan, J. F. (2001). The effects of a pneumatic tourniquet on blood loss in total knee arthroplasty. Canadian journal of Surgery, 44(1), 33.

16. Li, B., Wen, Y., Wu, H., Qian, Q., Lin, X., \& Zhao, H. (2009). The effect of tourniquet use on hidden blood loss in total knee arthroplasty. International orthopaedics, 33(5), 1263-1268.

17. Hou, D., Youssef, E. A. S., Brinton, T. J., Zhang, P., Rogers, P., Price, E. T., ... \& March, K. L. (2005). Radiolabeled cell distribution after intramyocardial, intracoronary, and interstitial retrograde coronary venous delivery: implications for current clinical trials. Circulation, 112(9_supplement), I-150.

18. Boswell, C. A., Ferl, G. Z., Mundo, E. E., Bumbaca, D., Schweiger, M. G., Theil, F. P., ... \& Khawli, L. A. (2011). Effects of anti-VEGF on predicted antibody biodistribution: roles of vascular volume, interstitial volume, and blood flow. PLOS One, 6(3).

19. Yuan, T., Cong, Y., Meng, J., Qian, H., Ye, W., Sun, W. S., ... \& Bao, N. R. (2017). Arachidonic acid causes hidden blood loss-like red blood cell damage through oxidative stress reactions. Journal of Surgical Research, 211, 14-20.

20. Yuan, T., Fan, W. B., Cong, Y., Xu, H. D., Li, C. J., Meng, J., ... \& Zhao, J. N. (2015). Linoleic acid induces red blood cells and hemoglobin damage via oxidative mechanism. International journal of clinical and experimental pathology, 8(5), 5044.

21. Qian, H., Yuan, T., Tong, J., Sun, W. S., Jin, J., Chen, W. X., ... \& Zhao, J. (2017). Antioxidants attenuate oxidative stress-induced hidden blood loss in rats. Turkish Journal of Hematology, 34(4), 334.

22. Saba Zaidi, S., \& Ishaque, N. (2017). Fat embolism syndrome, in a patient with Bilateral total knee replacement. Pakistan Journal of Neurological Sciences (PJNS), 12(2), 34-36.

23. Li, Q., Lin, Y., Wang, S., Zhang, L., \& Guo, L. (2017). GLP-1 inhibits high-glucose-induced oxidative injury of vascular endothelial cells. Scientific reports, 7(1), 1-9.

24. Nemes, R., Koltai, E., Taylor, A. W., Suzuki, K., Gyori, F., \& Radak, Z. (2018). Reactive oxygen and nitrogen species regulate key metabolic, anabolic, and catabolic pathways in skeletal muscle. Antioxidants, 7(7), 85.

25. Newsholme, P., Keane, K. N., Carlessi, R., \& Cruzat, V. (2019). Oxidative stress pathways in pancreatic $\beta$-cells and insulin-sensitive cells and tissues: importance to cell metabolism, function, and dysfunction. American Journal of Physiology-Cell Physiology, 317(3), C420-C433.

26. Chen, Q., Tang, L., Xin, G., Li, S., Ma, L., Xu, Y., ... \& Niu, H. (2019). Oxidative stress mediated by lipid metabolism contributes to high glucose-induced senescence in retinal pigment epithelium. Free Radical Biology and Medicine, 130, 48-58.

27. Subauste, A. R., \& Burant, C. F. (2007). Role of Fox01 in FFA-induced oxidative stress in adipocytes. American Journal of Physiology-Endocrinology and Metabolism, 293(1), E159-E164. 
28. Liu, W., Baker, R. D., Bhatia, T., Zhu, L., \& Baker, S. S. (2016). Pathogenesis of nonalcoholic steatohepatitis. Cellular and Molecular Life Sciences, 73(10), 1969-1987.

29. Gęgotek, A., \& Skrzydlewska, E. (2019). Biological effect of protein modifications by lipid peroxidation products. Chemistry and physics of lipids.

30. Wang, T., Deng, Y., Chen, Y., Qu, G., Feng, Z., Shang, J., ... \& He, N. (2019). Disordered Metabolism and Repair Mechanism: Mitochondria Influenced by Cationic and Neutral Nanoparticles. Journal of biomedical nanotechnology, 15(12), 2428-2438.

31. Gentile, F., Arcaro, A., Pizzimenti, S., Daga, M., Cetrangolo, G. P., Dianzani, C., ... \& Barrera, G. (2017). DNA damage by lipid peroxidation products: implications in cancer, inflammation and autoimmunity. AIMS genetics, 4(2), 103.

32. Ighodaro, O. M., \& Akinloye, O. A. (2018). First line defence antioxidants-superoxide dismutase (SOD), catalase (CAT) and glutathione peroxidase (GPX): Their fundamental role in the entire antioxidant defence grid. Alexandria Journal of Medicine, 54(4), 287-293.

33. Munne-Bosch, S., \& Pinto-Marijuan, M. (2016). Free radicals, oxidative stress and antioxidants. Encycl. Appl. Plant Sci, 2, 16-19.

34. Si, S., Li, L., Wang, Z., Wu, Y., Shan, G., Xu, B., ... \& Song, S. (2019). Cerium Oxide Nanoparticles Reduce X-ray Irradiation-Induced Damage to the Immune Cells by Upregulation of Superoxide Dismutase and Glutathione Peroxidase. Nanoscience and Nanotechnology Letters, 11(10), 14641469.

35. Liu, Y. H., Huang, Q. H., Wu, X., Wu, J. Z., Liang, J. L., Lin, G. S., ... \& Chen, J. N. (2018). Polydatin protects against acetaminophen-induced hepatotoxicity in mice via anti-oxidative and anti-apoptotic activities. Food \& function, 9(11), 5891-5902.

36. Qi, X., Qin, Z., Tang, J., Han, P., Xing, Q., Wang, K., ... \& Zhang, W. (2017). Omega-3 polyunsaturated fatty acids ameliorates testicular ischemia-reperfusion injury through the induction of Nrf2 and inhibition of NF-KB in rats. Experimental and molecular pathology, 103(1), 44-50.

37. Matough, F. A., Budin, S. B., Hamid, Z. A., Alwahaibi, N., \& Mohamed, J. (2012). The role of oxidative stress and antioxidants in diabetic complications. Sultan Qaboos University Medical Journal, 12(1), 5 .

38. Li, S., Tan, H. Y., Wang, N., Zhang, Z. J., Lao, L., Wong, C. W., \& Feng, Y. (2015). The role of oxidative stress and antioxidants in liver diseases. International journal of molecular sciences, 16(11), 2608726124.

\section{Figures}




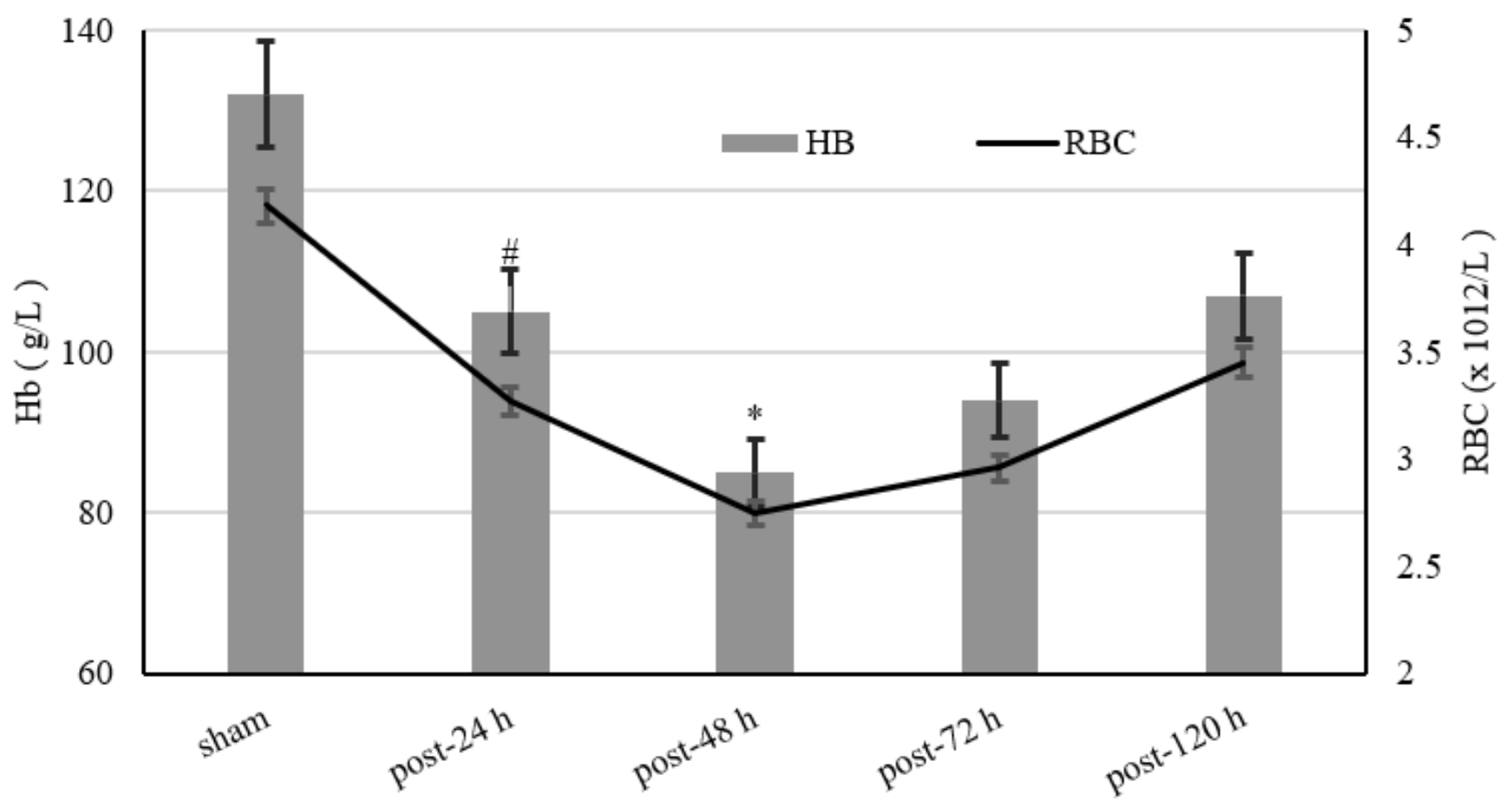

Figure 1

$\mathrm{Hb}$ and RBC changes before and after surgery. $\mathrm{Hb}$ and RBC decreased most significantly $48 \mathrm{~h}$ after operation. Values are shown as mean $\pm \mathrm{SE}$; ${ }^{*} \mathrm{P}<0.01$, $\# \mathrm{P}<0.05$
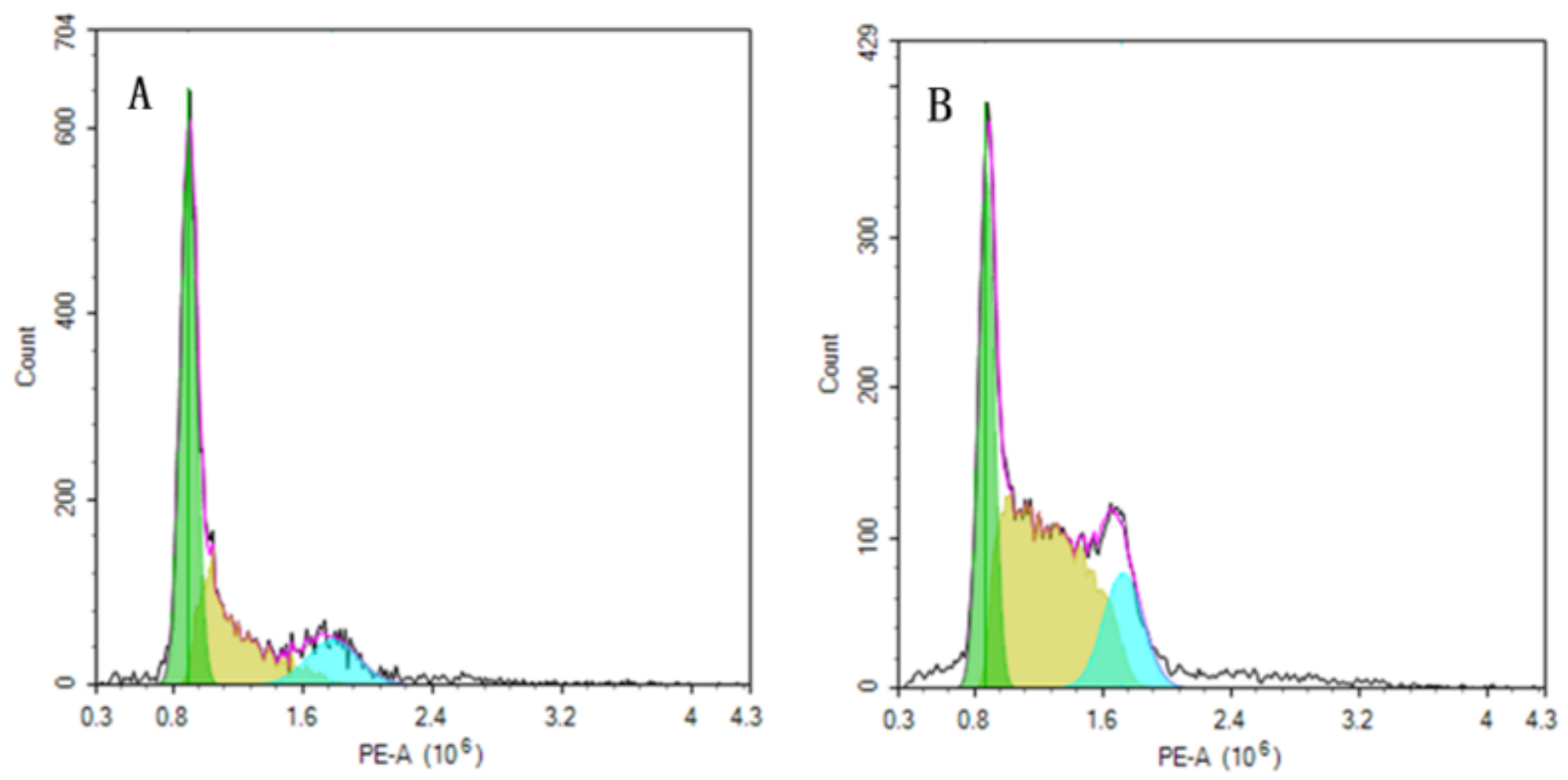

Figure 2 
ROS level changes pre-operation(A) and $48 \mathrm{~h}$ post-operation(B). The dyed cells were gathered to detect DCFH-DA fluorescence $(488 \mathrm{~nm}-552 \mathrm{~nm})$ using flow cytometry. Cell with fluorescence intensity at 102-103 were counted representing cells with high ROS release. The blue area represents the ROS level; The results showed that the ROS level at 48 hours after surgery was significantly higher than pre-operation; Meanwhile, HBL was the most obvious and FFA concentration reached the peak, suggesting that $\mathrm{HBL}$ is closely related to oxidative stress.

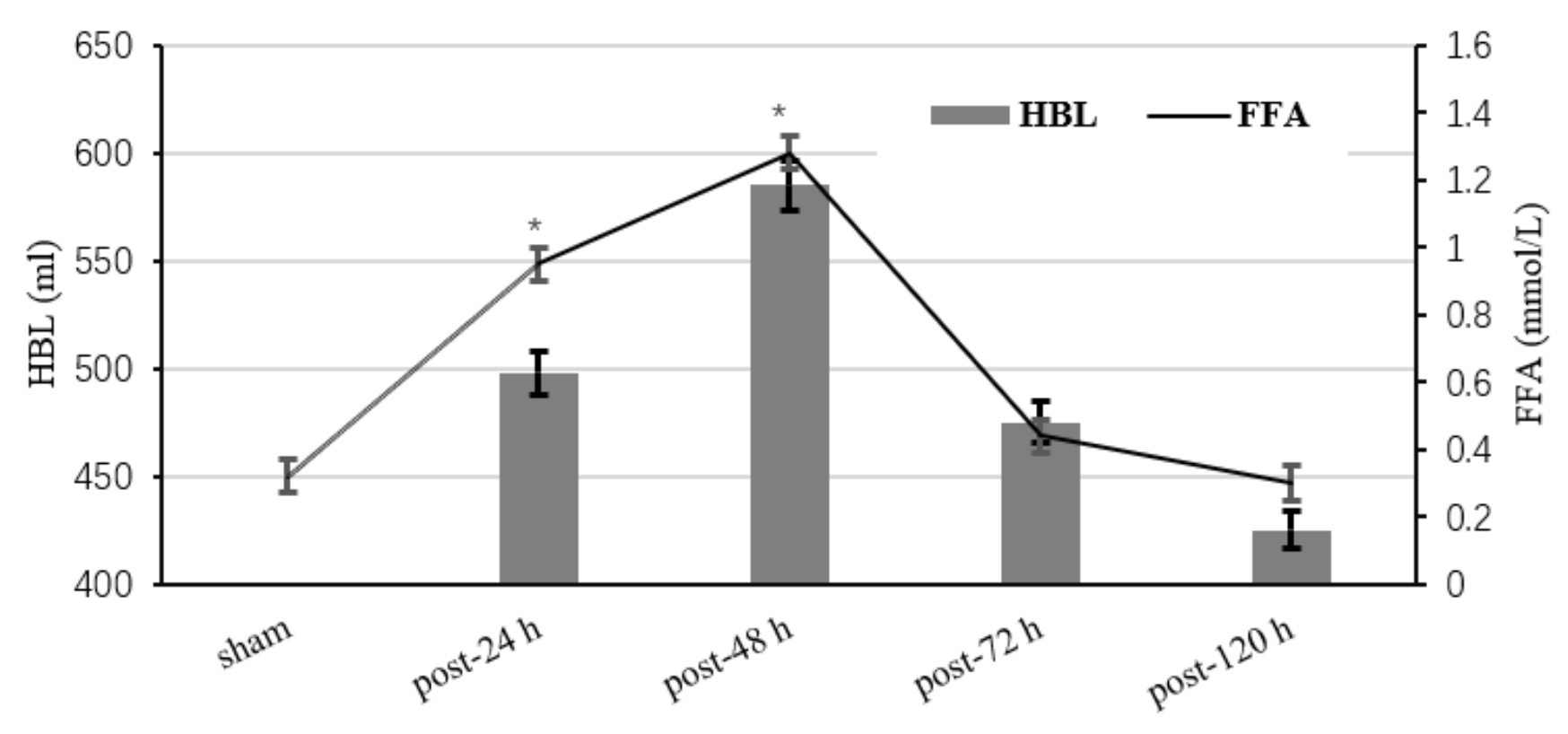

Figure 3

FFA and HBL changes before and after surgery. According to the variation tendency in the figure, $\mathrm{HBL}$ becomes more obvious with the increase of FFA concentration. Values are shown as mean $\pm \mathrm{SE}$; $* \mathrm{P}<$ $0.01, \# \mathrm{P}<0.05$ 

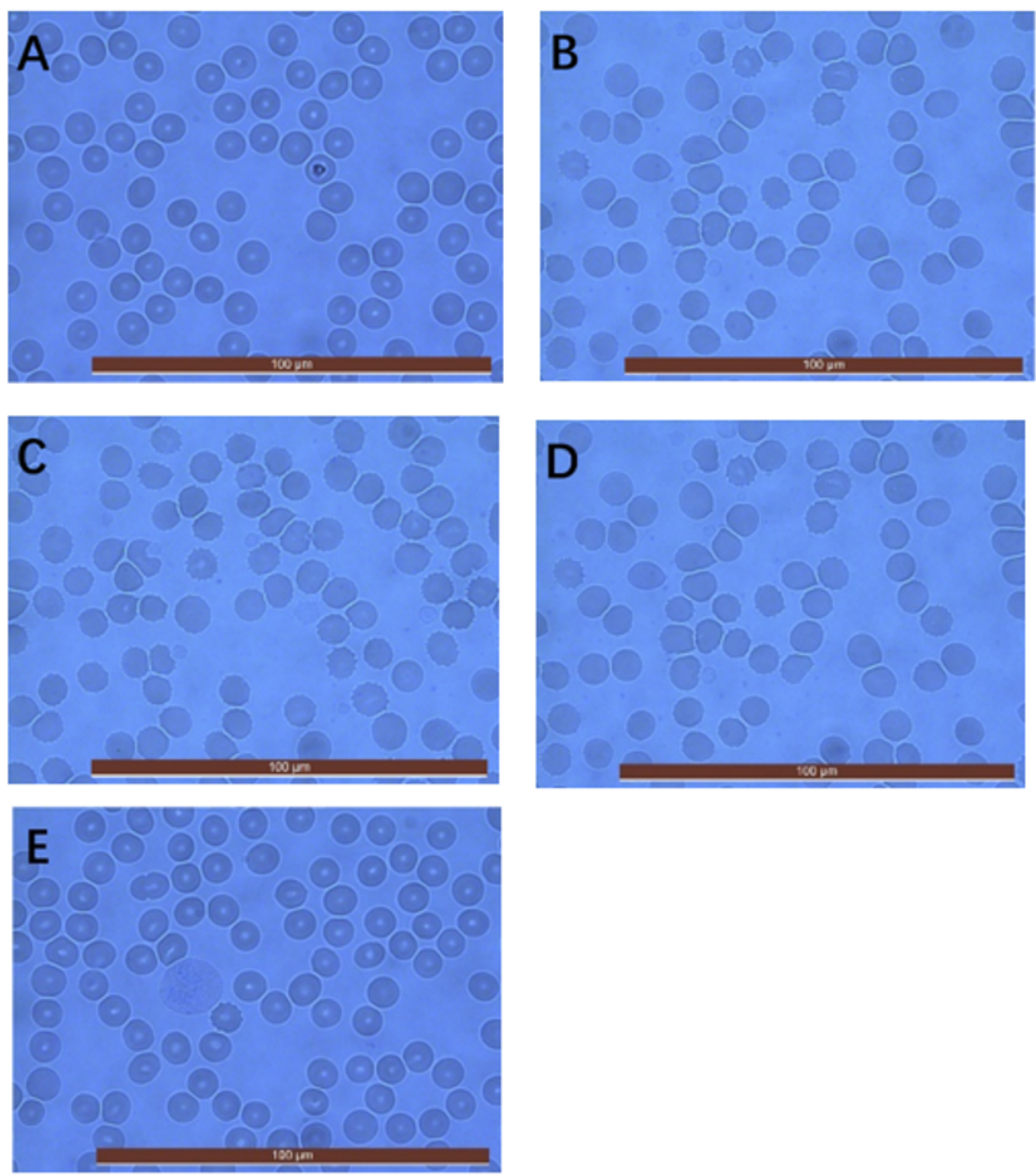

\section{Figure 4}

Morphological changes of red blood cells under microscope during perioperative period. Samples were

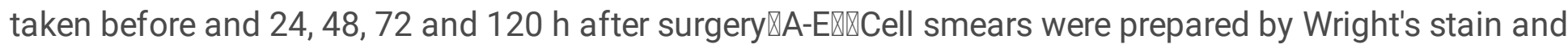
observed under polarized light microscope (400x magnification). A large number of atypical red blood cells can be found under the microscope at $24 \mathrm{~h}$ after operation (B). And $48 \mathrm{~h}$ after the operation, the atypia erythrocytes increased, mainly manifested by polymorphic changes, shrinkage, deformation, and 
even breaking (C); 72h later, the erythrocyte morphology improved, and the atypical cells decreased (D); However, the erythrocyte morphology was normal 5 days after the operation.

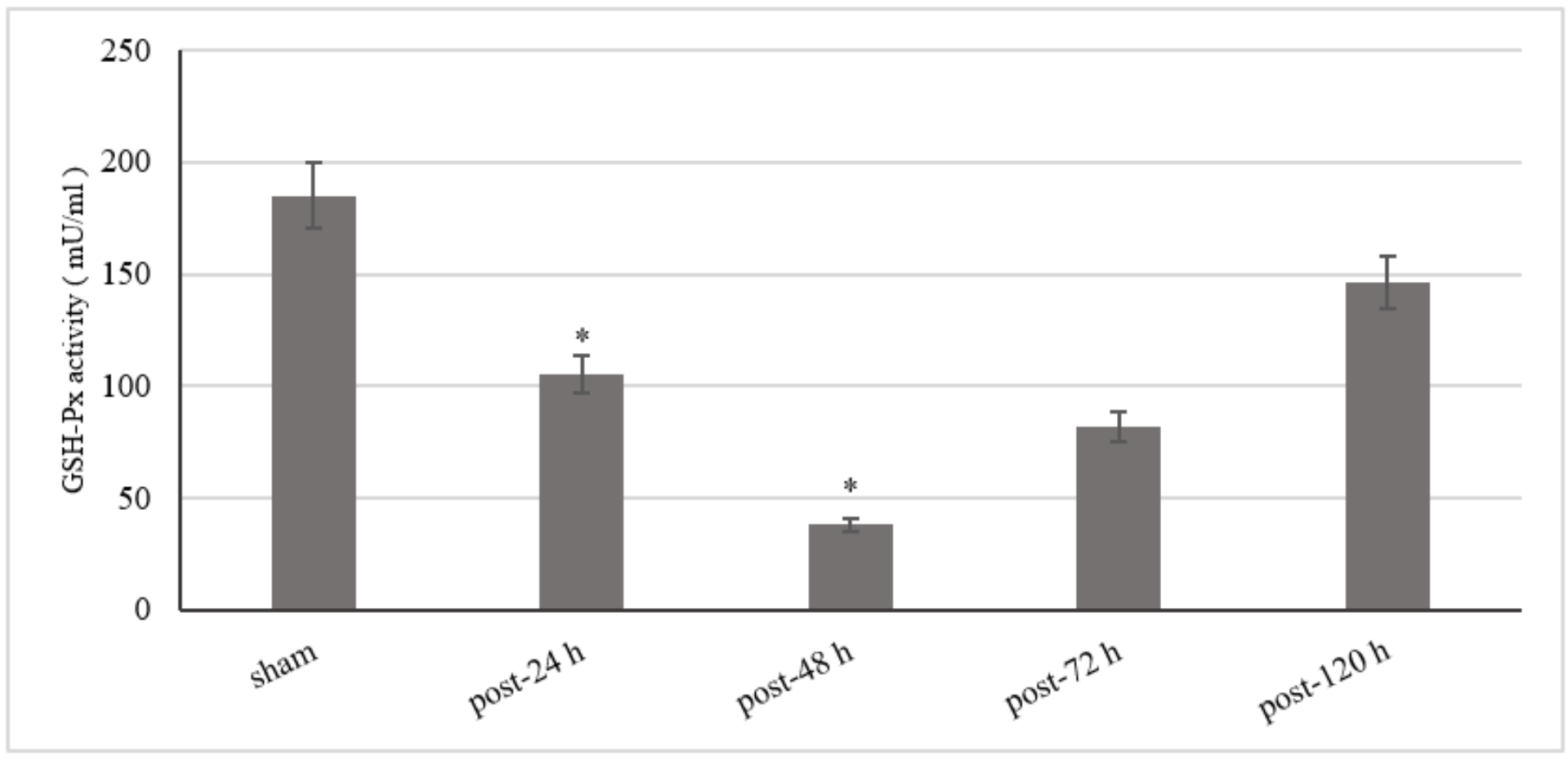

Figure 5

Changes of GSH-Px activity during perioperative period. Values are shown as mean $\pm \mathrm{SE}$; ${ }^{*}<<0.01$, \#P< 0.05

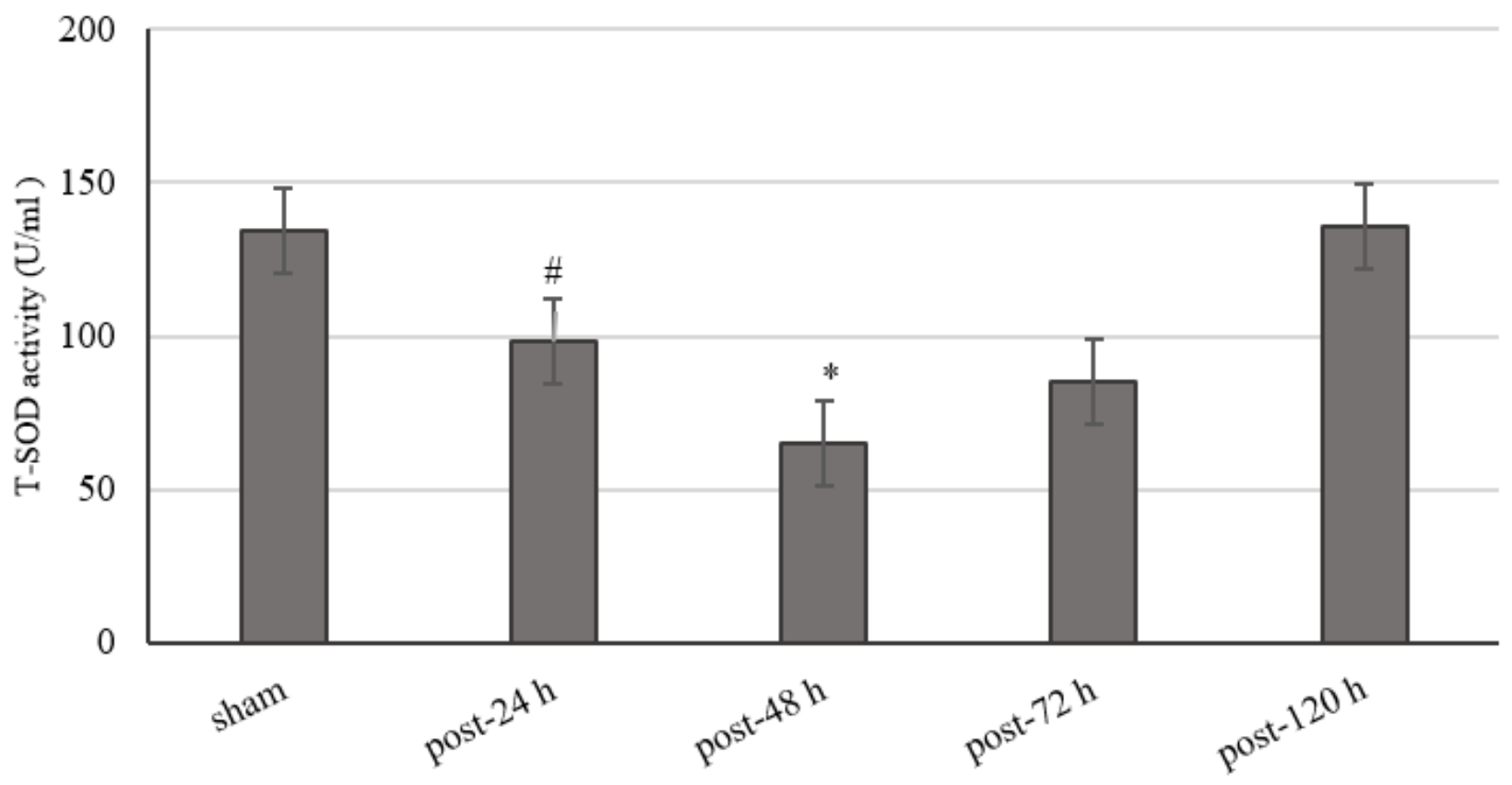

Figure 6 
Changes of T-SOD activity during perioperative period. Values are shown as mean $\pm \mathrm{SE} ;{ }^{*} \mathrm{P}<0.05, \# \mathrm{P}<$ 0.01

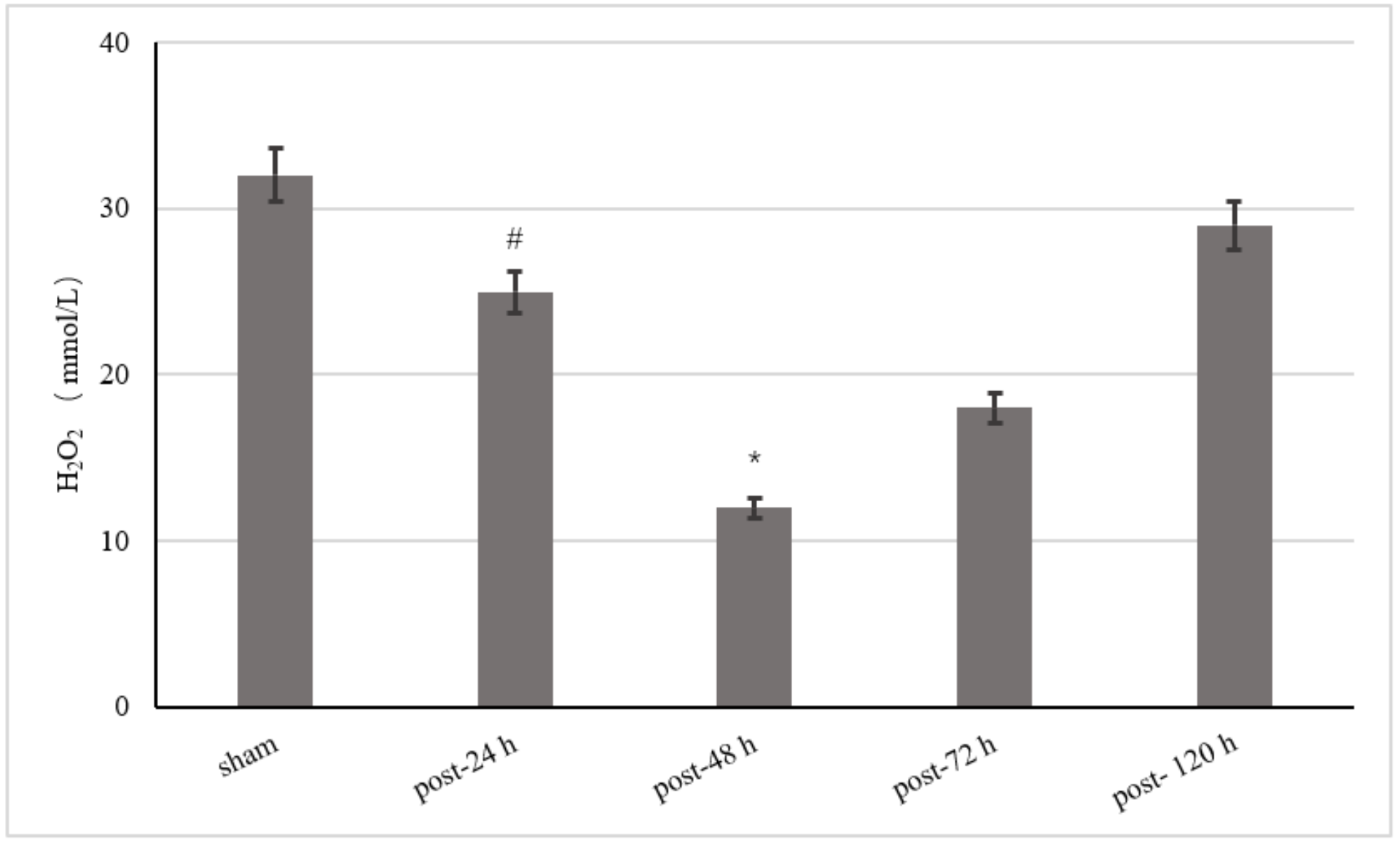

Figure 7

Changes of $\mathrm{H} 2 \mathrm{O} 2$ levels during perioperative period. Values are shown as mean $\pm \mathrm{SE} ;{ }^{*} \mathrm{P}<0.01, \mathrm{AP}<0.05$ 